and its precursor proBDNF in mood-stabilized patients with bipolar disorder: A study of two independent cohorts. J Affect Disorders 2014; 160: 1-9.

4 Tang YP, Wade J. Developmental changes in BDNF protein in the song control nuclei of zebra finches. Neuroscience 2013; 250: 578-87.

5 Li Z, Zhang Y, Wang Z, Chen J, Fan J, Guan Y, et al. The role of BDNF, NTRK2 gene and their interaction in development of treatment-resistant depression: Data from multicenter, prospective, longitudinal clinic practice. J Psychiatr Res 2013; 47: 8-14.

Chen Zhang, MD, PhD, Zezhi Li, MD, PhD, Yiru Fang, MD, PhD, Professor, Division of Mood Disorders, Shanghai Mental Health Center, Shanghai Jiao Tong University school of Medicine, Shanghai, China. Email: yirufang@gmail.com

doi: 10.1192/bjp.205.5.410a

\section{Early and delayed treatment of bipolar disorder}

Using Danish registry data, Kessing et al examined the relationship between lithium response and the timing of treatment (early $v$. delayed). ${ }^{1}$ Early treatment was associated with an increased probability of lithium response. This is a clinically important finding, given the increasing emphasis on early intervention in bipolar disorder. The results of the Kessing et al study are sobering. Only few patients, particularly among those for whom treatment was delayed, responded to lithium. Several factors may have contributed to the reported results.

The study did not - and possibly could not - control for the cycle shortening that is observed after successive episodes of bipolar disorder. Although the interpretation of such cycle shortening has been debated, ${ }^{2}$ it is well established that early cycles are significantly longer than those occurring later; consequently, early in the course of illness one would expect longer spontaneous remissions regardless of treatment. This effect may be partially responsible for the greater treatment response in patients receiving early intervention in the Kessing et al study.

Naturalistic studies typically demonstrate full response in about $30 \%$ of participants ${ }^{3}$ (that is, no recurrences, or the Kessing et al criterion, in treatment-adherent patients), which is markedly greater than the response rate observed by Kessing et al. This discrepancy could be related to age at first contact. The average age of participants whom Kessing et al reported as having received early and late treatment was 46.7 years and 49.1 years, respectively. The natural history of bipolar disorder includes an average age at onset in the second or third decade of life. The trajectory of the illness, where mania typically develops as the last stage, delays the diagnosis of bipolar disorder. Also, there is often a substantial delay in starting treatment even following the diagnosis of bipolar disorder. ${ }^{4,5}$ These reports, in conjunction with the advanced age at index presentation, and high rates of antidepressant, antipsychotic and anticonvulsant use in the Kessing et al study suggest that participants may have been afflicted with bipolar disorder for some time before 'first contact'. In a sample of 450 participants, Baldessarini et al reported a negative relationship between treatment latency and effect of treatment on time spent ill. ${ }^{5}$ If the aforementioned findings are generalisable to the Danish sample, the reduced overall treatment responses may be interpreted as a consequence of relatively advanced participant age.

Finally, Kessing et al analysed data collected since 1995. Is it possible that participants had received lithium during the years prior? This would further complicate the interpretations of sample responsiveness to lithium, regardless of early or late initiation. In conclusion, we suggest that the findings presented by Kessing et al are limited by the lack of control for inter-participant differences in the manifestation of the natural history of bipolar disorder. Such control may be difficult, or in some cases impossible, to achieve using registry-based observational data, but is nevertheless imperative to understanding the effects of early $v$. late treatment prophylaxis in relapsing-remitting illnesses such as bipolar disorder.
1 Kessing LV, Vradi E, Andersen PK. Starting lithium prophylaxis early v. late in bipolar disorder. Br J Psychiatry 2014; 205: 214-20.

2 Oepen G, Baldessarini RJ, Salvatore P, Slater E. On the periodicity of manicdepressive insanity, by Eliot Slater (1938): translated excerpts and commentary. J Affect Disord 2004; 78: 1-9.

3 Garnham J, Munro A, Slaney C, MacDougall M, Passmore M, Duffy A, et al. Prophylactic treatment response in bipolar disorder: Results of a naturalistic observation study. J Affect Disord 2007; 104: 185-90.

4 Ortiz A, Bradler K, Slaney C, Garnham J, Ruzickova M, O'Donovan C, et al. An admixture analysis of the age at index episodes in bipolar disorder. Psychiatr Res 2011; 188: 34-9.

5 Baldessarini RJ, Tondo L, Hennen J. Treatment-latency and previous episodes: relationships to pretreatment morbidity and response to maintenance treatment in bipolar I and II disorders. Bipolar Disord 2003; 5: 169-79.

Abraham Nunes, MD, MBA, Tomas Hajek, MD, PhD, Martin Alda, MD, Department of Psychiatry, Dalhousie University, Halifax, Nova Scotia, Canada. Email: malda@dal.ca

doi: $10.1192 /$ bjp.205.5.411

Authors' reply: We are confident that the relatively low response rates to lithium in our study relate to the narrow definition of lithium response, rather than to characteristics of the included patients. ${ }^{1}$ Thus, we intended to characterise patients who had an excellent response to lithium monotherapy; that is, patients who were 'cured' from further affective episodes following a start-up period of lithium as in a prior study. ${ }^{2}$ We used two robust clinical indicators to define excellent lithium response: (a) lithium prescribed in monotherapy; and (b) no need for psychiatric hospital admission. By doing this, we defined lithium response in a rather rigorous way, resulting in relatively low rates of response. We do not find that our definition of lithium response hampered the finding of the study that early treatment with lithium was associated with increased probability of excellent lithium response compared with delayed treatment, or hampered the generalisability of this finding. Although cycle acceleration occurs on average in bipolar disorder ${ }^{3,4}$ the results of our study may suggest that early treatment with lithium might prevent progression of bipolar disorder.

1 Kessing LV, Vradi E, Andersen PK. Starting lithium prophylaxis early v. late in bipolar disorder. Br J Psychiatry 2014; 205: 214-20.

2 Kessing LV, Hellmund G, Andersen PK. Predictors of excellent response to lithium: results from a nationwide register-based study. Int Clin Psychopharmacol 2011; 26: 323-8.

3 Kessing LV, Hansen MG, Andersen PK. Course of illness in depressive and bipolar disorders. Naturalistic study, 1994-1999. Br J Psychiatry 2004; 185 372-7.

4 Kessing LV, Olsen EW, Andersen PK. Recurrence in affective disorder: analyses with frailty models. Am J Epidemiol 1999; 149: 404-11.

Lars Vedel Kessing, Psychiatric Center Copenhagen, Department $O$ and Faculty of Health and Medical Sciences, University of Copenhagen, Per Kragh Andersen Department of Biostatistics, University of Copenhagen, Denmark. Email: Department of Biostatistics, Un, Uedel.Kessing@regionh.dk
Lars.Ver

doi: 10.1192/bjp.205.5.411a

\section{'Reasonable adjustments' for vulnerable patients}

We support the views of Tuffrey-Wijne \& Hollins ${ }^{1}$ and their argument for the NHS to take an organisational approach to embed documentation and provision of reasonable adjustments for those with protected characteristics under the Equalities Act 2010. Lord Darzi defined quality for the NHS as comprising three dimensions: safety, effectiveness and patient experience. ${ }^{2}$ The provision of reasonable adjustments is central to each of these.

Safety - Tuffrey Wijne \& Hollins rightly identify the lack of provision of reasonable adjustments as being a patient safety issue. The Confidential Inquiry into Premature Deaths of People with 